\title{
An update on pain
}

T. Renton ${ }^{1}$

Professor Renton will be giving 'An update on pain' on Saturday 3 May at the 2008 British Dental Conference and Exhibition, held at the Manchester Central Convention Complex.

Pain is a personal, subjective experience that involves sensory, emotional and behavioural factors. Most of us at some stage in our lives have experienced pain. The most common type of pain that springs to mind is the acute or 'healthy' inflammatory pain associated with a bee sting or toothache. But increasingly, in the western world, one in three of us will also experience chronic pain, or unhealthy 'nerve' pain, most commonly associated with backache, headaches or persistent post-surgical pain. As this is a persistent state and derives from the nerves themselves, it is significantly more of a challenge to treat. This article highlights some significant progress made in the assessment and treatment of patients with neuropathic pain. An improved understanding of chronic or neuropathic pain by practitioners and patients themselves, will undoubtably better equip those involved to recognise and manage the pain.

\section{What is pain?}

Pain is a personal, subjective experience that involves sensory, emotional and behavioural factors associated with actual or potential tissue injury. It is defined by the International Association for the Study of Pain as 'An unpleasant sensory and emotional experience associated with actual or potential tissue damage or described in terms of such damage.' ${ }^{1}$

Most of us at some stage in our lives have experienced pain. The most common type of pain that springs to mind is the acute or inflammatory pain associated with a bee sting or toothache. Most of us have felt the emotional, non-physical pain associated with watching a loved one experience physical or emotional trauma. Indeed, in the western world, one in three of us will also experience chronic pain most commonly associated with backache, headaches or persistent post-surgical pain, costing the USA alone over $\$ 100$ million in lost income and insurance costs. ${ }^{2}$ So in essence there

\footnotetext{
${ }^{1}$ Professor of Oral Surgery, Department of Oral Surgery, Dental Institute, King's College London, King's Denmark Hill Campus, Bessemer Road, London, SE5 9RW Correspondence to: Professor Tara Renton Email: tara.renton@kcl.ac.uk
}

DOI: 10.1038/bdj.2008.201 are many types of pain that can vary in their perceived intensity, character, duration, frequency, site, associated disability and emotional impact.

It is well known that the perception of pain will vary due to many factors, for example: gender, age, ethnicity and pain experience. Soldiers working in a war zone will have a much higher pain threshold than the rest of us. It is also acknowledged that stress and anxiety will significantly reduce one's pain threshold. Pain itself causes anxiety and depression thus compounding the unpleasant experience. In essence pain is a combination of physical sensation with a significant emotional and cognitive component, which is reflected by the parts of the brain activated during pain stimulus as seen during functional MRI scanning (Fig. 1).

Only the patient can experience their pain and unfortunately due to our limited vocabulary, description or expression of the pain experience is often severely compromised. Thus assessment of pain is often difficult and subjective. As dentists we are the unsung heroes of acute pain management and anxiety in our patients. We consistently see patients whom, without masochistic tendencies, would rather be anywhere

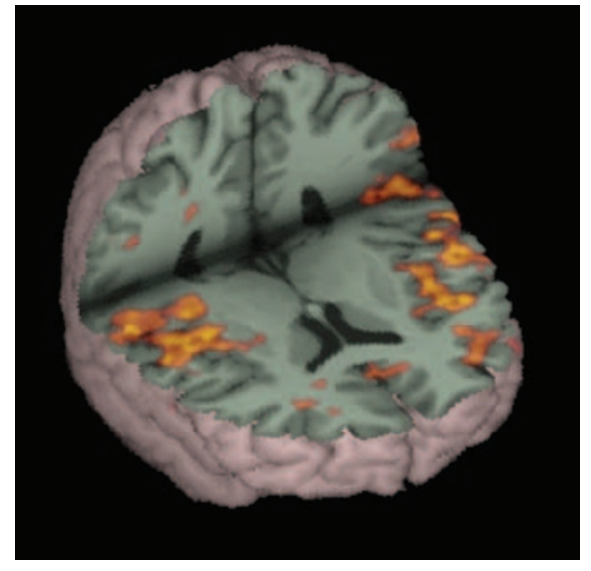

Fig. 1 Functional MRI image showing changes in blood flow to areas of the brain responding to a painful stimulus in a conscious patient

else aside from the dental chair undergoing injections and dental treatment. Our profession is unique in that we are trained as undergraduates in exposure prone techniques with competency in local analgesia and patient management in stressful conditions during dental treatment. Most medical students learn these skills as postgraduates.

\section{So what has changed in our under- standing of acute or chronic pain?}

The onset of the 21st century has been an incredibly exciting time in pain biology. 
Recent studies in basic pain research have revealed numerous novel targets for the advent of new pain therapies. Major advances range from molecular studies that have identified transduction proteins in pain neurons, and genetic variation in pain modulation, to cortical imaging studies, which reveal how pain is experienced on a cognitive level. ${ }^{2,3}$

Pain is induced by tissue damage that results in cell death and subsequent release of intracellular factors (proteases) causing activation of the phospholipid pathway, the kinin pathway, mast cells releasing histamine and platelet aggregation, resulting in serotonin release. These factors initiate depolarisation of local pain responsive nerve fibres (A delta fibres display pain and cold sensitivity and $\mathrm{C}$ fibres display warm and pain sensitivity) via newly discovered pain receptors or ion channels. The evoked potential is relayed through primary to secondary neurons (in the dorsal horn of the spinal cord for most sensory neurons but within the trigeminal nucleus for the trigeminal sensory fibres), ultimately evoking tertiary neuron activation in the cortex. This not only results in the sensation of pain and a physical response (withdrawal from the noxious stimulus) but a downward neural response that causes nerveinduced inflammation at the injured site. Neuroinflammation causes the bulk of the inflammatory response at the injured site. Different types of neurons, receptors, channels and transmitters have been identified in the peripheral versus central neural pathways, improving our understanding of the mechanisms of existing analgesics.

\section{The two main types of pain}

Healthy acute 'nociceptive pain' (sprains, bone fractures, burns, bumps, bruises, inflammation [from an infection or arthritic disorder])

Nociceptors are the nerves which sense and respond to parts of the body which suffer from damage. The pain is typically well localised, constant, and often with an aching or throbbing quality. Visceral pain is the subtype of nociceptive pain that involves the internal organs. It tends to be episodic and poorly localised due to the slow conduction by $\mathrm{C}$ fibres.
Characteristics include resolution of pain when irritant stimulus is removed and inflammation subsides, and that it tends to respond well to treatment with opioids and anti-inflammatory drugs.

Unhealthy chronic 'neuropathic pain' or nerve pain (post-herpetic neuralgia, reflex sympathetic dystrophy/causalgia [nerve trauma], components of cancer pain, phantom limb pain, entrapment neuropathy [eg, carpal tunnel syndrome], and peripheral neuropathy)

Peripheral neuropathy is alarmingly prevalent in the UK and diabetes is the most common cause (diabetic burning foot). Other causes include chronic alcohol use, exposure to other toxins (including many chemotherapies), vitamin deficiencies, HIV infection and a large variety of other medical conditions. Chronic pain is defined as pain lasting longer than three months, or pain that is persistent after the cause of inflammation is no longer present. This chronic state involves complex neuroplastic mechanisms including pain memory, sensitisation and potentiation by either central, peripheral or a combination of both components of the nervous system. This process is essentially understood to be a disease of the nervous system and is characterised by lack of response to opioids or non-steroidal anti-inflammatory drugs (NSAIDs). The efficacy of gabapentin, the 5\% lidocaine patch, tramadol hydrochloride and tricyclic antidepressants (TCAs) has been consistently demonstrated in multiple randomised controlled trials. ${ }^{4}$ Thus acute and chronic pain are separate conditions requiring differing strategies in assessment and management.

\section{New techniques in the measure- ment and assessment of pain}

Functional magnetic resonance imaging (fMRI) is a system whereby a conscious pain can be stimulated (noxious, olfactory, visual, auditory) and the changes in blood supply to areas of the brain can be identified by differentiation of oxygenated versus deoxygenated blood (Fig. 1). This technology has revolutionised central pain pathway activity in man and to some extent has replaced animal experiments.

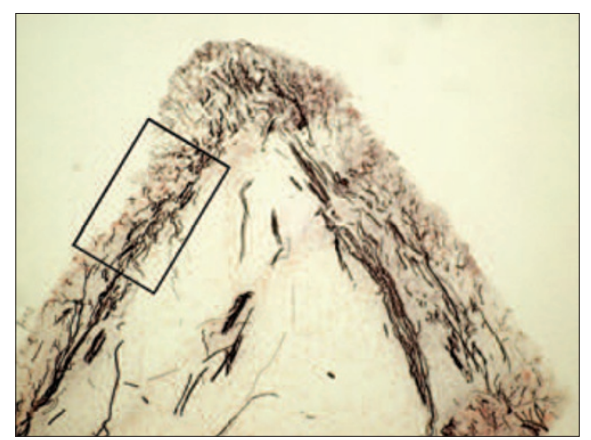

Fig. 2a A photomicrograph illustrating the neurofilament immunoreactive fibre staining of a control dental pulp revealing the small pain-related nerve fibres in the subodontoblastic plexus. Magnification $\times 20$

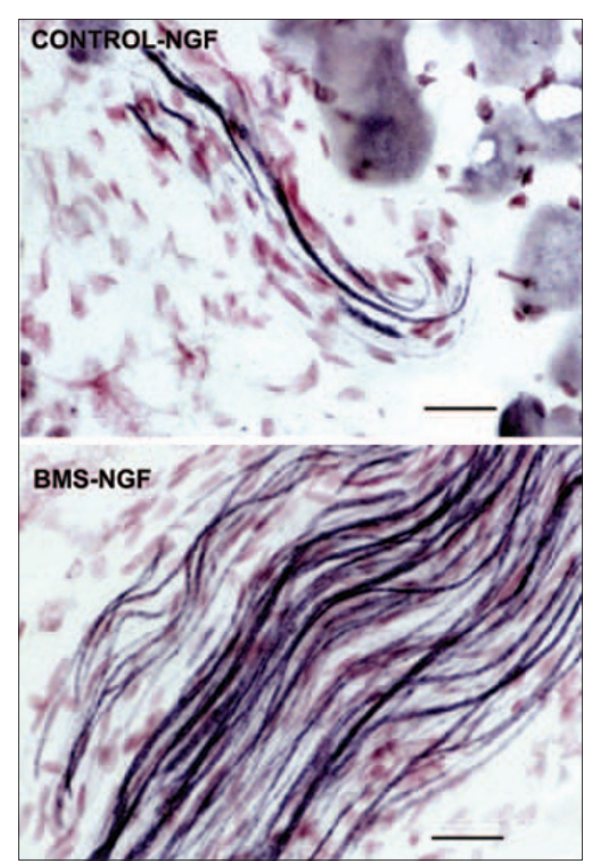

Fig. 2b Nerve growth factor immunoreactive fibre staining of a control tongue (top panel) and a burning mouth syndrome tongue (bottom panel), revealing increased pain active fibres in BMS. Magnification $\times 40$

Researchers have reported that in chronic pain conditions, the patient's reported visual analogue scale (VAS) correlates with the brain vascular activity, thus providing us with a potentially objective 'in vivo' pain measure in man.

Laboratory techniques have also assisted us in visualising pain 'down the microscope'. By using immunohistochemistry we can label specific pain receptors on nerve tissue in biopsies harvested after assessing the patient. Commonly in neuropathic pain there is degeneration of the peripheral nociceptors and relative increase in expression of pain channel receptors (diabetic pain, vulvadynia, irritable bowel syndrome, breast pain). ${ }^{5}$ This technique has been applied to 
toothache (Fig. 2a) and the phenomenon of increased pain channels has also been reported in burning mouth syndrome (Fig. 2b). Calcium channel imaging also allows the researcher to evaluate in vivo cellular activity when challenged with a known receptor adjuvant.

Modality sensory testing is used to assess the activity of the pain-specific nerve fibres. A delta fibres respond to cooling and pain and C fibres respond to warming and pain. By using cooling and warming stimuli, hyperactivity can be identified and recorded objectively. This form of testing comprises part of a group of tests referred to as quantitative sensory testing, which is gaining popularity in the assessment of neuropathic pain.

Genetics of pain. It is proposed that approximately 30,000 genes of the human genome might be involved in pain mediation. As previously stated in this article, several sodium channels have been identified as key players in neuropathic pain. There are some familial conditions whereby certain sodium ion channels are absent, rendering those affected unable to feel pain, resulting in considerable tissue damage. Genes associated with sodium channel expression include SCN1A, discovered to be cause of familial hemiplegic migraine (FHM), and SCN9A in neuropathic pain as well as in inability to experience pain. Other genes have also been identified in modulating pain, including GTP cyclohydrolase (GCH1), which modulates sensitivity to pain in normal individuals and liability to chronic pain, and catechol-0-methyltransferase (COMT) and the cytochrome P450 variant allele CYP3A5, which modulate the genetic response to opioid medications in humans.

By combining clinical and laboratory methods of assessment, translational or 'bed to bench' research is unravelling and will continue to unravel the complex mechanisms leading to pain. There are already over 50 phase 1 trials worldwide, assessing specific pain receptor blocking agents, a novel and potentially revolutionary method of preventing pain. Thanks to recent developments, we clinicians are infinitely better equipped to counsel our patients in our understanding of the mechanisms of their pain, which undoubtedly assists the patient in coming to terms with their pain. Overall, one can remain optimistic that over the next decade significant developments will minimise patient suffering due to pain.

\section{Trigeminal pain}

The trigeminal (three twins) is a sensory nerve which takes up the bulk of the sensory cortex of the human mind. It is no wonder that when we experience pain from the three regions supplied by this sensory nerve, we can think of little else. Dentists are trained well in the causes of healthy acute inflammatory pain, but less well in chronic pain. Medical students have minimal exposure to supraclavicular pain attributable to dental or ENT conditions and their main exposure is to trigeminal neuralgia during their neurosurgical rotation. It is due to these phenomena that chronic trigeminal pain often falls within the 'pavement cracks' between different specialists, often resulting in conflicting advice to longsuffering patients seeking relief from their suffering.

There are many attempts in classifying orofacial pain. The main categories are topographical (odontogenic versus non-odontogenic) and chronic versus acute. A recent cluster analysis ${ }^{6}$ (Fig. 3) has revolutionised the way many of

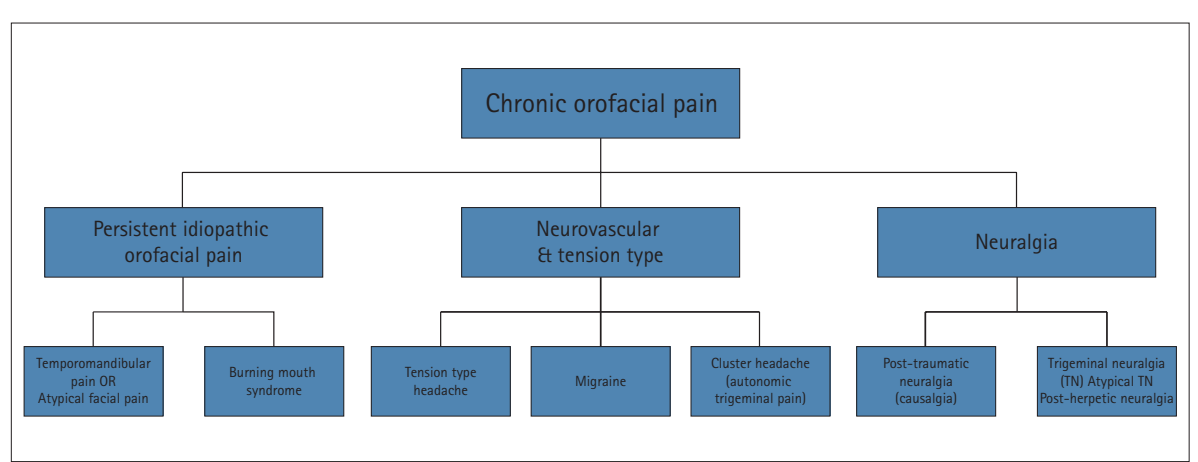

Fig. 3 Cluster analysis classification of chronic orofacial pain us perceive chronic orofacial pain. The neurovascular pain is predominantly V1 or ophthalmic division pain, comprising mainly of headaches and giant cell arteritis. The new phenomenon of medical overuse headache is not included in this analysis and is an increasing problem in the western world, requiring three months of 'cold turkey' therapy before effective treatment of the original headache can be initiated.

The neuralgia group includes trigeminal neuralgia (typical and atypical; always bare in mind patients under 40 years must have multiple sclerosis excluded), post-herpetic neuralgia (postshingles pain, which is $60 \%$ increased likelihood if the patient is over 50 years of age) and post-traumatic neuralgia (causalgia, commonly seen in post-operative cases [breast surgery 30\% of cases, hernia surgery $30 \%$ of cases, spinal surgery, and knee surgery $20 \%$ of cases]). The idiopathic group includes burning mouth syndrome, which increasingly is believed to be a small fibre neuropathy (much like burning foot in diabetic neuropathy), although as yet the cause is unknown.

Temporomandibular joint pain is a complex problem involving possible muscle or ligamentous strains, cartilage misplacement or damage, arthritides, pathology and psychometric elements. Pain in the joints is often present without any clinical or radiographic evidence of mechanical dysfunction. In contradiction, mechanical and anatomical dysfunction is also commonly present with no associated pain. This heterogenous group of patients provide the practitioner with plenty of difficulties in management but most respond to simple reversible interventions, for example giving up chewing gum, or simple lower soft splints and reassurance.

The outlying conditions outwith this cluster analysis included persistent idiopathic orofacial pain (atypical facial pain, atypical odontalgia). Some believe that this group contains patients with causlagia, as many patients' chronic pain is initiated with dental surgical intervention. They may present with persistent post-traumatic pain due to peripheral sensory nerve branch damage caused by local anaesthesia or surgery. They are a 
particularly difficult group of patients to treat, often presenting with whole quadrants of extracted teeth, having undergone hours of dental restorative treatment resulting in multiple extractions with no relief from the pain.

There is increasing evidence for specific psychological and medical management of many of these chronic orofacial pain conditions. It is imperative to spend time listening to the patient, taking an exhaustive history, thorough examination and an awareness of up-to-date assessment methods. Evaluating these patients requires time and patience and often a multidisciplinary team. Often this investment of one-on-one assessment will itself empower the patient to understand and cope with their pain.

The author wishes to acknowledge the following individuals: Professor Praveen Anand, Department of Peripheral Neuropathy, Imperial College London; Professor Steve Williams and Dr Matthew Howard, Department of Clinical Neuroscience, Institute of Psychiatry and Psychology, King's College London; and Dr Sheelah Harrison, Lead Clinician, Orofacial Pain Centre, King's College London Dental Institute.
1. Pain terms: a list with definitions and notes on usage. Recommended by the IASP Subcommittee on Taxonomy. Pain 1979; 6: 249.

2. Woolf C J, Salter M W. Neuronal plasticity: increasing the gain in pain. Science 2000; 288: 1765-1769.

3. Hunt S P, Mantyh P W. The molecular dynamics of pain control. Nat Rev Neurosci 2001; 2: 83-91.

4. Dworkin R H, Backonja M, Rowbotham M C et al. Advances in neuropathic pain: diagnosis, mechanisms, and treatment recommendations. Arch Neurol 2003; 60: 1524-1534.

5. Facer P, Casula M A, Smith G D et al. Differential expression of the capsaicin receptor TRPV1 and related novel receptors TRPV3, TRPV4 and TRPM8 in normal human tissues and changes in traumatic and diabetic neuropathy. BMC Neuro/ 2007; 7: 11.

6. Woda A, Tubert-Jeannin S, Bouhassira D et al. Towards a new taxonomy of idiopathic orofacial pain. Pain 2005; 116: 396-406.

\section{Erratum}

\section{Access to dental services in Scotland: an analysis of dentist-population ratios (BDJ 2008; 204: E6)}

It has been brought to our attention that the above online research paper contains the following errors: in Table 2, column 3, the entry for 'Greater Glasgow' should be 540, not 54.00 as printed; in Table 3, column 1, the Words 'Highland' and 'Greater Glasgow' should be transposed to correspond to the correct rows of figures. The authors and editors wish to apologise to $B D J$ readers for any confusion caused by these errors. 